\title{
A New Open Curve Detection Algorithm for Extracting the Laser Lines on the Road
}

\author{
Huijie Fan ${ }^{1,2, a}$, Lipo Zhao ${ }^{1,2, b}$, Siyuan $\mathrm{He}^{1,2, \mathrm{c}}$ and Yandong Tang ${ }^{2, \mathrm{~d}}$ \\ ${ }^{1}$ State Key Laboratory of Robotics, Shenyang Institute of Automation, Chinese Academy of \\ Science, Shenyang 110016, China \\ ${ }^{2}$ Graduate University of the Chinese Academy of Science, Beijing 100049, China \\ afanhuijie@sia.cn, bzhaolipo@sia.cn, chesiyuan@sia.cn, dytang@sia.cn
}

Keywords: road surface roughness, open curve, line enhance filtering, snake model, edge inhibit operator

\begin{abstract}
This paper proposes a new open curve detection algorithm for recognizing the laser rays on the road to verify the roughness of the road surface by parsing the curvature of the laser rays. The laser line in the image is first enhanced by the bright line enhance filtering to remove the clutters generated by the background. The enhanced line has the characteristics that its gray value is greater than that of background, so the established energy functional in open curve detection process has a gray value constraint to drive the evolution curve to the brightest place in image. The line on the road surface is an open curve throughout the whole image, so we fixed the two endpoints of the evolution curve to maintain it is an open curve in curve evolution. Moreover, we design an edge inhibit operator to restrain the influence of objects edges to ensure the accuracy of the test laser line. We compared our algorithm with the edge detection operators, and the comparison results showed that our method is more accurate and more robustness to the background.
\end{abstract}

\section{Intorduction}

Road surface roughness is an important indicator in road surfacing, construction acceptance and evaluation of urban road surface. It directly determines the vehicle's comfort and road safety. Traditional methods [1,2] of pavement roughness detection mainly rely on manual collection, and they have the disadvantages of high subjectivity, low efficiency and safety problems. In the past few years, with the development of information technology and automatic controlling, micro-measurement technology has advanced greatly in precision, and this makes the pavement roughness measurement accuracy can fully meet the requirements of high-grade highways, airport runways and so on. The more advanced methods of pavement roughness measurement have adopted the laser as the light source. They use a high-speed vehicle-mounted digital camera system to continually collect the road surface images, and then identify the road surface roughness automatically by means of image processing.

Fig. 1 shows the process of road surface roughness measurement. It is based on the technology of structured light detection. Line laser is used as structured light. Planar CCD camera with a high frame rate can image transverse sections of road surface which are illuminated by laser.

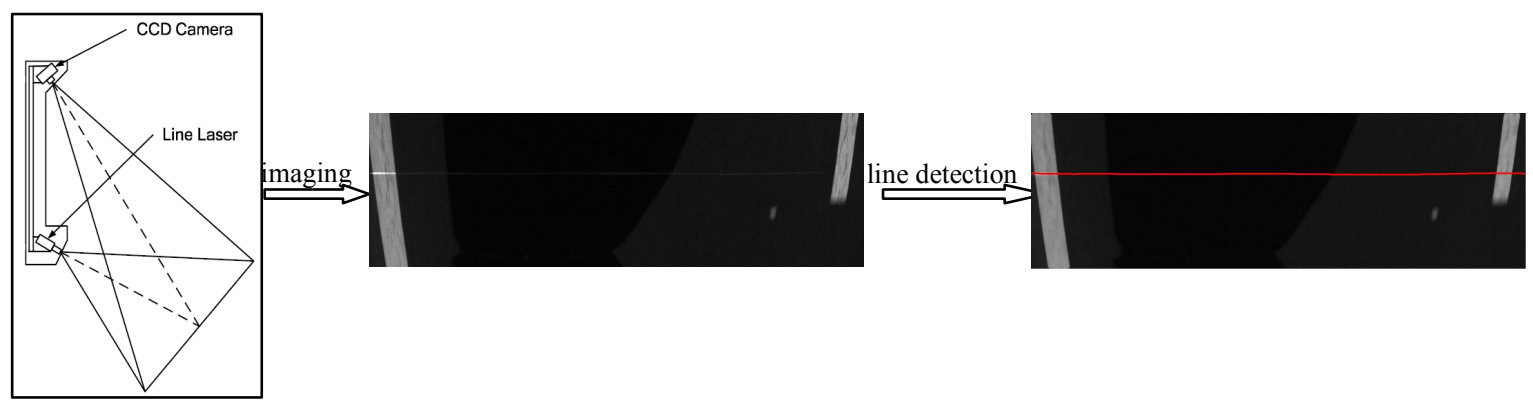

Fig. 1. The process of road surface roughness measurement 
We must first extract the laser line in the image and remove the outliers, and a number of image processing techniques have been used to detect the laser line from the image. The widely used tools for segmenting the laser line are always based on edge detection operators. The method in Ref. [3] uses intensity histograms of the images, so it cannot detect the line part similar to the background and it cannot handle the complex background images. Chang et al. use adaptive threshold [4] set by intensity histograms of the images to segment the laser line. The adaptive threshold value can be also set by weighted neighborhood average [5] rather than intensities. Nonetheless the edge detection operators are not always effective for the noisy images with low contrast and strong noise. They still suffer from the problems of determining the correct global or local thresholds in processing the intensity inhomogeneous images automatically, and these methods always need to link the detected discontinuous segments and falsely extraction results always occur due to the noise influence. In this paper, we propose a new open curve detection algorithm via the line enhance filtering $[6,7]$ and the curve evolution method [8] based on the imaging characteristics of the laser line projected onto the road surface. The laser line in the image is enhanced by a second order of Gaussian filtering. The enhanced line has the characteristics that its gray value is greater than that of background, so the established energy functional in curve evolution process has a gray value constraint to drive the evolution curve to the brightest place in image. The line on the road is an open curve throughout the whole image, so we fixed the two endpoints of the evolution curve to maintain it is an open curve in curve evolution. Moreover, we design an edge inhibit operator to restrain the influence of objects edges to ensure the accuracy of the target laser line.

\section{Open Curve Detection Model}

\subsection{Bright Line Enhance}

\subsubsection{Second Derivative of Gaussian Filter}

The laser transmitter installed in the bottom of the vehicle, therefore the laser line projected onto the road surface has different intensity in the vehicle's shadow and non-shaded areas. The laser line is not very clear in the road marking area (shown in Fig. 1), too. Therefore, we first enhance the laser line by means of the bright line enhance filtering.

Traditional line detectors response not only to lines but also to edges, for instance, they will give high responses to the edges of bright lines or blobs when we only want to detect bright lines. This will cause false detections in many images. A 2D Gaussian filtering is widely used in the area of image processing. Generally it performs low pass filtering. A 2D Gaussian filtering with standard deviation $\sigma$ is defined in Formula (1) and its plot is shown in Fig. 2a. Its first and second derivatives are also widely used. For example, the line detection technique proposed by [6]. Our enhanced method is inspired by the line detection method proposed in [6], and the difference is that we aim at detecting the bright lines in image. We use the second order derivative of Gaussian filter to enhance the bright line. It is defined in Formula (2) and its appearance is shown in Fig. 2b. Fig. 2c shows the second derivative of Gaussian represented in 2D space.

$$
\begin{aligned}
& \mathrm{G}_{\sigma}(x, y)=\frac{1}{\sqrt{2 \pi} \sigma} \mathrm{e}^{-\frac{x^{2}+y^{2}}{2 \sigma^{2}}} . \\
& \mathrm{G}_{\sigma}^{\prime \prime}(x, y)=\frac{\left(\sigma^{2}-x^{2}\right)\left(\sigma^{2}-y^{2}\right)}{2 \pi \sigma^{10}} \mathrm{e}^{-\frac{x^{2}+y^{2}}{2 \sigma^{2}}} .
\end{aligned}
$$




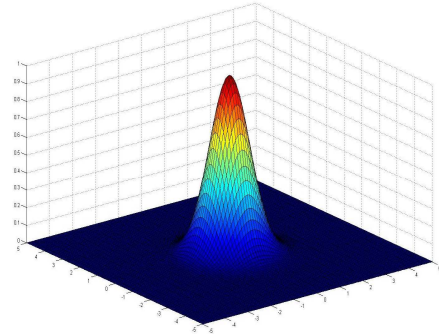

a)

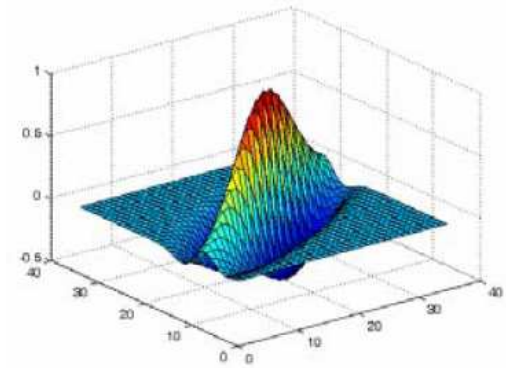

b)

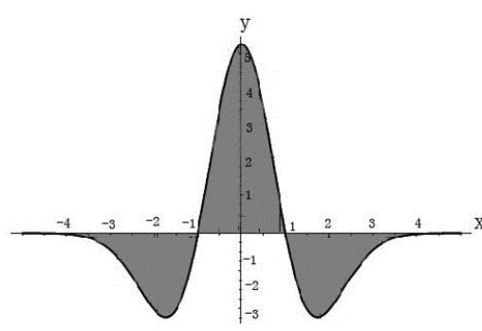

c)

Fig. 2. Appearance of filters. a) Gaussian filter. b) second derivative of Gaussian filter.

c) second derivative of Gaussian represented in 2D space.

An experiment is done to show the advantages of the line enhancement filtering, as shown in Fig. 3. Fig.3b e are the enhanced results of four different edge operators. Fig. $3 \mathrm{f}$ is the result of our method. We can see that our enhancement algorithm can effectively remove the clutters generated by the background and the laser line enhanced.

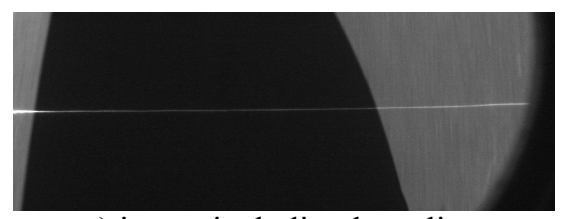

a) image including laser line

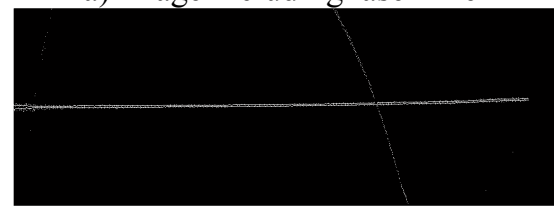

d) enhanced by Sobel operator

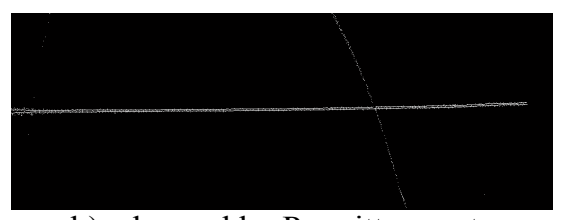

b)enhanced by Prewitt operator

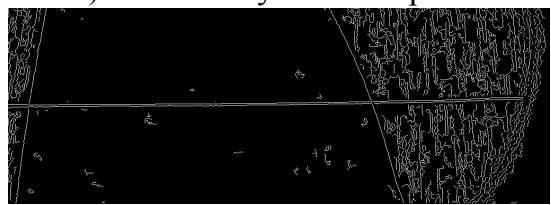

e) enhanced by Canny operator

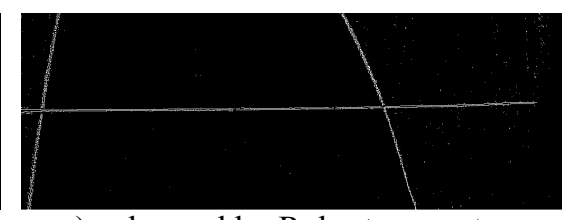

c) enhanced by Roberts operator

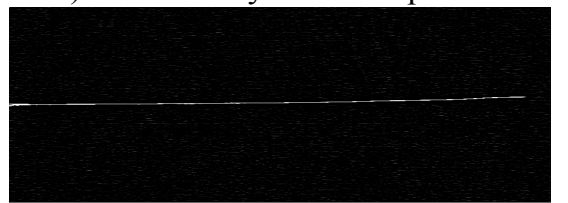

f) enhanced by our method

Fig. 3. Comparison results of different methods.

\subsubsection{Edge Inhibit Operator}

In experiments we found that, sometime the bright objects on the pavement and shadows project onto the white road markings will generate some bright edges. The line enhance filtering will enhance the laser line and meanwhile the bright interference edges. Figure $5 \mathrm{~b}$ shows the enhanced results of Figure 5a. We can see that the enhanced interference edges are clear and close to the laser line. These would disturb the following curve evolution process and finally get the wrong results (Fig. 5c).

We propose an edge inhibit operator $R$ to effectively suppress the bright interference edges according to the different characteristics of the target line and the interference edges. $R=e^{-\left|c_{1}-c_{2}\right|}$, and $c_{1}$ and $c_{2}$ are the mean value of the region upper and lower part of the template respectively. Fig. 4 represent the principle of the edge inhibit operator. If the template center, which is current point, is located at the linear area (Fig. 4a), $c_{1} \approx c_{2}$, thus $R=e^{-\left|c_{1}-c_{2}\right|}$ is large and $R$ will strengthen the enhance results of the current point.

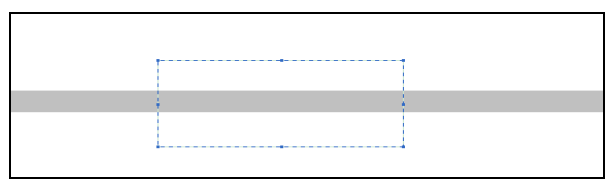

a) template center is located at linear area
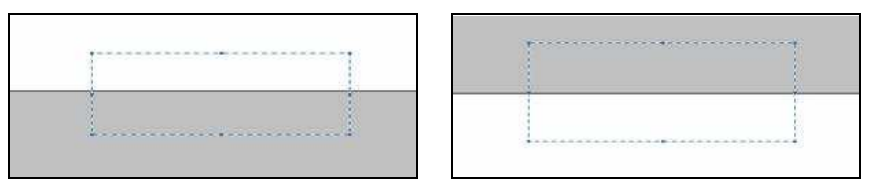

b) template center is located at the edges

Fig. 4. The edge inhibit operator 


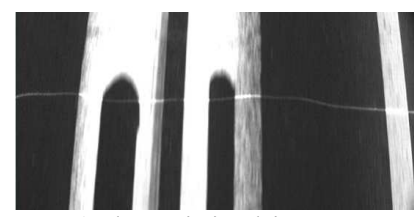

a) the original image

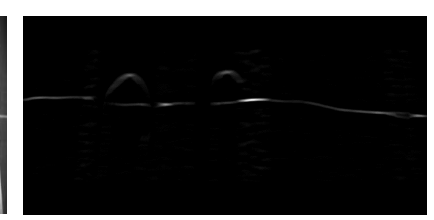

b) the enhanced results

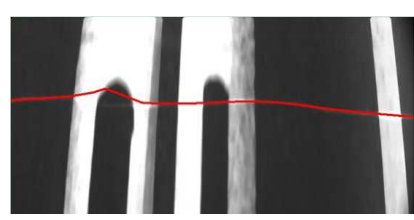

c) no edge inhibit operator

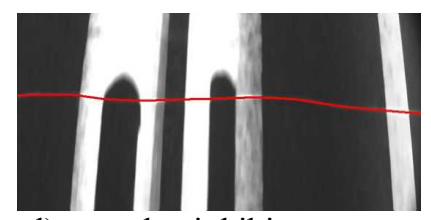

d) use edge inhibit operator

Fig. 5. Comparison results of using the edge inhibit operator

If the template center is located at the edges (Fig. 4b), $c_{1} \neq c_{2}$, thus $R=e^{-\left|c_{1}-c_{2}\right|}$ is small and $R$ will weak the enhance results of the current point. Fig. 5c and Fig.5d show the comparison results before and after adding the edge inhibit operator. We can see that the edge inhibit operator can overcome the impact from the enhanced non-target edges and guide the curve move to the correct results.

\subsection{Open Curve Evolution Model}

\subsubsection{Open curve representation}

Let $\Omega$ be a bounded open subset of $R^{2}$ and $C$ be an evolving curve denoting the boundary of the open subset $\omega$ of $\Omega$ (i.e. $C=\partial \omega$ ). Let $I: \Omega \rightarrow$ R be a given image. Our method uses B-spline curve for open curves approximation (for more details, please refer to [8]). Let $\mathrm{P}_{i}(i=0,1, \ldots, n)$ be the $n+1$ consecutive control points. The $k$-degree B-spline curve $C(s)$ can be defined by:

$$
C(s)=\sum_{i=0}^{n} P_{i} N_{i, k}(s) \text {. }
$$

where $N_{i, k}(s)$ is the harmonic functions. In accordance with the recursive formula, $N_{i, k}(s)$ can be defined as:

$$
N_{i, k}(s)=\frac{s-t_{i}}{t_{i+k-1}-t_{i}} N_{i, k-1}(s)+\frac{t_{i+k}}{t_{i+k}-t_{i+1}} N_{i+1, k-1}(s) .
$$

where $t_{i}(i=0,1, \ldots, n)$ are the nodal values, and they constitute the knot vector of the $k$-degree B-spline function. If $s \in\left[t_{i}, t_{i+1}\right), N_{i, 1}=1$; else, $N_{i, 1}=0$.

In this paper, we use 3-degree B-spline function to represent the evolution curve. First, it is a smooth curve, and then it has a continuous second derivative. Its matrix representation is:

$$
C(s)=\frac{1}{6}\left[s^{3}, s^{2}, s, 1\right]\left[\begin{array}{cccc}
-1 & 3 & -3 & 1 \\
3 & -6 & 3 & 0 \\
-3 & 0 & 3 & 0 \\
0 & 4 & 1 & 0
\end{array}\right] \times\left[\begin{array}{l}
P_{i-1} \\
P_{i} \\
P_{i+1} \\
P_{i+2}
\end{array}\right] .
$$

where $s \in[0,1]$.

\subsubsection{Open Curve Evolution}

Kass et al. proposed the curve evolution model in 1987 [9]. The snake model has been widely used in image segmentation problems. It uses curve evolution process to detect the target from a given image. First of all, an initial curve $C$ is given, and then an energy functional is obtained according to image data and target features. Finally, the target is achieved by minimizing the energy functional along with the curve deformation.

Let $y=f(x), x \in[o, w]$ be a curve in image. where $w$ is the value of image width. We define energy functional $E$ according to the target characteristics in image, and get the curve evolution function by minimizing the energy functional $E$. Then give the initial curve extracted from the enhanced image, the evolution curve will finally move to the laser line we aims to extract. The laser line in the enhanced image has the characteristic that it is a bright line, and it is almost smooth. The energy functional can be defined as follow: 


$$
E(f)=\int_{0}^{w} \sqrt{1+f^{\prime}(x)^{2}} d x+\int_{0}^{w} \frac{1}{1+\lambda \cdot u(x, f(x))^{2}} d x .
$$

where $u(x, f(x))$ is the gray value at the point $(x, f(x))$, and $\lambda$ is the non-negative parameter. The first term is called the regularization term which measures the smoothness of the evolution curve. The last term of $E$ is called the data fitting term which drives the evolution curve move to the bright area.

Parameterizing the gradient descent direction by an artificial time $t \geqslant 0$, the Euler-Lagrange equation can be derived by minimizing the energy functional $E$ in (6) with respect to $\varphi$ :

$$
\frac{\partial \varphi(x, t)}{\partial t}=\frac{d}{d x}\left(\frac{1}{\sqrt{1+\left(\frac{\partial \varphi(x, t)}{\partial x}\right)^{2}}} \frac{\partial \varphi(x, t)}{\partial x}\right)+2 \lambda \frac{u(x, \varphi(x, t)) \cdot u_{y}(x, \varphi(x, t))}{\left(1+\lambda \cdot u(x, \varphi(x, t))^{2}\right)^{2}} .
$$

The boundary conditions are:

$$
\frac{\partial \varphi(0, t)}{\partial x}=0 \quad \text { and } \quad \frac{\partial \varphi(w, t)}{\partial x}=0 .
$$

The initial condition is:

$$
\varphi(x, 0)=\phi(x) .
$$

where $y=\phi(x)$ is the initial curve.

The finite difference method is used to discretize the partial differential equation in (7) for the numerical solution of $\varphi(x, t)$. Let $h$ be the space step, $\Delta t$ be the time step, $x_{i}=i h$ be the grid points for $1 \leqslant i \leqslant w$, and $\varphi_{i}^{n}=\varphi\left(x_{i}, t_{n}\right)=\varphi(i h, n \Delta t)$ is an approximation of $\varphi(x, t)$ with $n \geqslant 0$. The finite differences are:

$$
\Delta_{-} \varphi_{i}=\varphi_{i}-\varphi_{i-1}, \quad \Delta_{+} \varphi_{i}=\varphi_{i+1}-\varphi_{i} .
$$

The discrete form of partial differential equation in (7) is:

$$
\frac{\varphi_{i}^{n+1}-\varphi_{i}^{n}}{\Delta \mathrm{t}}=\frac{\varphi_{i+1}^{n}-\varphi_{i}^{n}}{\sqrt{1+\left(\varphi_{i+1}^{n}-\varphi_{i}^{n}\right)^{2}}}-\frac{\varphi_{i}^{n}-\varphi_{i-1}^{n}}{\sqrt{1+\left(\varphi_{i}^{n}-\varphi_{i-1}^{n}\right)^{2}}}+2 \lambda \frac{u\left(i, \varphi_{i}^{n}\right) \cdot u_{y}\left(i, \varphi_{i}^{n}\right)}{\left(1+\lambda \cdot u\left(i, \varphi_{i}^{n}\right)^{2}\right)^{2}} .
$$

where the non-negative parameters $\Delta t=0.1$ and $\lambda=0.1$ are fixed in all experiments. The curve evolution will stop when the steady solution of the discrete function is acquired under the stop criterion: $\max _{i}\left|\varphi_{i}^{n+1}-\varphi_{i}^{n}\right|<0.01$.

\section{Experiment results}

We test our algorithm on detecting laser lines from different kinds of images. Experimental results are compared with the edge detection operator. We use canny edge detection method on the enhanced image, and then connect the detected edges as the final line detection results. All experiments are done in MATLAB, and the experimental environment is Windows XP, Intel core $2.8 \mathrm{GHz}$ CPU and $1 \mathrm{G}$ RAM.

Fig. 6 shows the experiment results on different kinds of images. The first row is the results of the edge detection operator, and the second row is the results of the proposed method. From the comparison results we can see, our algorithm can get the correct and smooth laser lines from various kinds of background. While the edge detection operator cannot detect the exact laser lines if the lines are projected on bright background, where there are no gradient. For example, the result in the upper left image misses the right part of laser line. Moreover, from the middle left and bottom left images, we can see that the edge detection methods are easy to be impact by interference edges. 

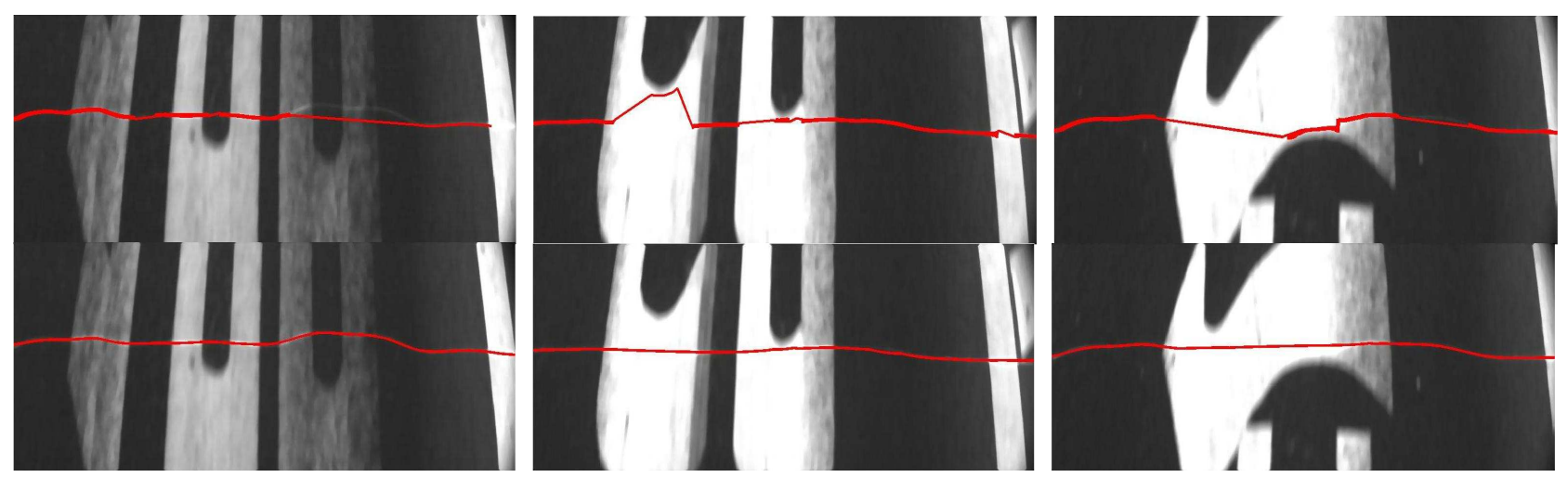

Fig. 6. Comparison results of using the edge inhibit operator. The first row is the results of canny edge detection, the second row is the results of our methods

\section{Conclusion}

In this paper, we propose a new open curve detection method to detect the laser line from images. Different from edge detection operator algorithms and popular line detection methods, our algorithm utilizes the image data and the target features to establish the energy functional and drive the initial curve to the laser line. We compared our algorithm with the canny-based edge detection method on a variety of background images. The experiment results show that the proposed model can exactly detect the laser line.

\section{Acknowledgements}

This work has been funded by Natural Science Foundation of China under Grant No. 50876110.

\section{References}

[1] Lu Z.X., Zhao L.Y., Li X.Q. and Yuan J., Simulation of Road Surface Roughness Based on the Piecewise Fractal Function, International Federation for Information Processing 2011, pp. 294-305.

[2] Youngguk Seo, Development and Implementation of Korea's First Percent within Limit (PWL) Specification for Road Pavements, Journal of Civil Engineering (2010), 14(3): pp. 353-361.

[3] Kirschke K.R. and Velinsky S., A histogram-based approach for automated pavement-cracks sensing, Journal of Transportation Engineering, ASCE, New York, NY, 1992, 119(3): pp.700-710.

[4] Chang H D, Shi X J and Glazier C, Real-Time Image Thresholding Based on Sample Space Reduction and Interpolation Approach, Journal of Computing in Civil Engineering, ASCE/October, 2003.

[5] Xu B.G. and Huang Y.X., Development ofan Automatic Pavement Surface Distress Inspection System, Final Report, October,2003.

[6] Li Q., Zhang L., You J., Zhang D. and Bhattacharya P., Dark line detection with line width extraction, 15th IEEE International Conference on Image Processing ,ICIP 2008. San Diego, CA, 12-15 October, 2008, pp. 621- 624.

[7] Keokanlaya S., Somsak C., Shatoshi W., and Kazuhiko H., Fingerprint Image Enhancement with Second Derivative Gaussian Filter and Directional Wavelet Transform, 2010 Second International Conference on Computer Engineering and Applications, pp. 112-116.

[8] Li X.M., Zhu L.L. and Tang Y.D., Boundary Detection Using Open Spline Curve Based on Mumford-Shah Model, ACTA AUTOMATICA SINICA, 2009. Vol. 35, No. 2. pp. 132-136.

[9] M. Kass, A. Witkin, and D. Terzopoulos, "Snakes: Active contour models," International Journal of Computer Vision, vol. 1, pp. 321-331, 1987. 